\title{
The "tract" of history in the treatment of lumbar degenerative disc disease
}

\author{
Khalil J. Chedid And Mokbel K. Chedid, M.D. \\ University of Michigan, Ann Arbor, and Henry Ford Health System and Oakwood Health System, \\ Dearborn, Michigan
}

\begin{abstract}
In this paper past, present, and future treatments of degenerative disc disease (DDD) of the lumbar spine are outlined in a straight forward manner. This is done to review previous knowledge of the disease, define current treatment procedures, and discuss future perspectives. An analysis of a subject of this magnitude dictates that one describes as accurate a history as possible: an anatomical/historical "tract" with emphasis on all possible deviations.

Although spinal disorders have been recognized for a long time, the view of DDD as a particular disease entity is a more recent development. In this paper, the authors attempt to outline the history of DDD of the lumbar spine in an unbiased and scientific fashion. Physiological, diagnostic, and therapeutic implications will all be addressed in this study.
\end{abstract}

KEY WORDS - degenerative disc disease - lumbar spine - history of surgery

The search for cures to health ailments most likely dates to the beginning of human history. Treatment of spinal disorders follows a similar path. A review of early Egyptian (1550 BC), Greek, Roman, and Arabic texts on medicine reveals an ongoing interest in treating spinal disorders. Sciatica and low-back pain were perhaps first mentioned by Hippocrates and later by Galen. ${ }^{10}$

Degenerative disc disease of the lumbar spine was not recognized as a specific disease entity until the last century and most work in this area was undertaken during the last quarter of that century. The surgical treatment of discogenic sciatica, as we know it today, was not realistically established until 1933, when it was first described by Mixter and Barr, although earlier attempts were present. Aggressive spine surgery, as is presently used, did not flourish until the 1970s. ${ }^{5,10-13,17}$

The last century furnished an abundance of information on spinal conditions that will open new gateways for more precise treatments in the future. In the past, treatment of disorders of the spinal column was focused on fractures and trauma, with some attention to low-back pain. Surgical treatment of disc ailments has progressed slowly. In fact four distinct stages of treatment of DDD seem to have occurred, especially in the last century.

Stage I involved the correct diagnosis of the problem and the selection of surgical treatment such as excision. In

Abbreviations used in this paper: $\mathrm{CT}=$ computerized tomography; $\mathrm{DDD}=$ degenerative disc disease $\mathrm{MR}=$ magnetic resonance .
Stage II advances were made in diagnostic methods, our understanding of physiological and biomechanical aspects of the disc, and treatment (methods of stabilization, types of instrumentation, methods of fusion, and so forth). Stage III consists of a period during which both Stages I and II continue with new trends of disc replacement by prosthetic devices (this stage has begun, but not been fully completed). In Stage IV, the previous three stages continue to progress together with a combination of the three along with more aggressive research into the field of disc degeneration and regeneration and applicable treatments.

Parallel to the aforementioned four stages, one should remember the importance of research in establishing the causes of degeneration and an understanding of the mechanics involved in DDD so that a more effective treatment can be devised. In this paper we will attempt to dissect the aforementioned stages, tracing the history of the disease. ${ }^{2,7,20,22}$

\section{THE TRACT OF HISTORY IN DDD}

Early studies in the treatment of spinal disorders focused primarily on trauma, as in Egypt ca. 1550 BC. Hippocrates (460-377 BC) may have been the first to describe spinal conditions in a scientific manner and to apply specific treatments. Many physicians followed the teachings of Hippocrates, but it was not until the seventh century that surgery was first suggested for spinal column injury by Paulus of Aegina. ${ }^{4,10,13,15,18}$ 
The earliest methodical and research-oriented study of spinal disorders seems to have been undertaken by Galen during the second century AD. He left behind inspiring treatises regarding anatomy and diseases of the spine..$^{12}$ Galen recognized lordosis, kyphosis, scoliosis, and succussion, and may have been the first physician to describe back pain. Many other physicians later added to the knowledge provided by Galen, a bit here and a bit there. Most of our information on the treatment of the spine before the Arabic era came from translations of Galen and others into Arabic between 700 and 900 . For many centuries, Arabic medicine was the basis for many medical advances. Albucasis (936-1013) left behind a huge medical encyclopedia, containing several neurosurgery volumes (Al Tasrif), and Avicenna (981-1037) provided us with his notable book, The Canons of Medicine. The influence of the Arabic schools continued through the Renaissance. During this period, some progress was made along with several advances in medicine. ${ }^{10,15}$

In relation to the spine, one should not forget a treatise published by the notable Turkish physician Sabuncuöglu in the 15th century. This physician was born in 1385 in Amasya, Turkey, and enlightened physicians and scientists by a description of his treatment of spinal disorders in his treatise The Imperial Surgery. Sabuncuöglu treated sciatica with medical and heat cauterization. During this period of history, low-back pain was still considered to be a traumatic injury and, if it became intractable, was treated with cauterization if analgesic agents had failed. The Imperial Surgery was, and still is, a landmark text given that medical progress was relatively idle during the 12th and 13th centuries. The concept of DDD, as we understand today, was not clearly identified. ${ }^{2,15}$

As mentioned earlier, most spine treatments focused on injuries. In the seventh century, Paulus of Aegina proposed the operative repair of spinal column injuries. Between 1510 and 1590 Ambroise Paré and Michel Mercatus used methods of suspension to align spine fractures, which were similar to a method used in earlier times. In Germany Johannes Schultheis (1595-1645) described another apparatus for the treatment of spinal injuries. The shift to the performance of surgery was slow. John Bell (1763-1820) expressed his doubts about surgical treatment. The spine was ultimately approached with trephination in 1814. Sir Percival Pott (1713-1788) operated on the spine for tuberculosis. Infections were the greatest enemy with which surgeons had to contend. ${ }^{10,13}$

The works of Lister (1882-1912), as well as others before him, on fighting infections made surgery more feasible. Surgical procedures continued to be performed. MacEwen (1848-1924) described the laminectomy procedure and Ménard (1895-1934) described a costotransversectomy approach. During the same time period, investigators such as Weber (1827), Rauber (1876), and Messerer (1880) began to perform some research studies of the biomechanics of the spine. Then, in 1895, William Conrad Röntgen (1845-1923) established imaging and the evolution of spinal disorders entered a new path. At first only anteroposterior x-ray views were available until Davis obtained a lateral radiograph in 1925. Another developmental milestone was met around 1930 with the introduction of myelography; this imaging modality later helped Dan- dy and others to remove intervertebral dises via laminectomies. In the US, however, A.G. Smith was the first to perform a laminectomy in $1829.4,10,14,16$

The lumbar disc did not become a center of attention until 1933, when W. J. Mixter and J. S. Barr provided the correct pathogenesis of disc herniation and, hence, offered an appropriate surgical treatment. The neurogenic nature of sciatica had first been described by Domenico Cotugno (1764). Following him, several other French neurologists such as Lasegue, Dejerine, and Sicard enriched our understanding of the basis of sciatica. The work of the German pathologists Schmorl and Andrea (1927-1929) established the modern basis for understanding the intervertebral disc, by providing very clear discussions of herniations as well as degenerations. . $^{2,4,5,16}$

Surgery for disc herniation was first performed by Oppenheim and Kruse (1909); later Mixter and Barr performed the laminectomy via the transdural approach to remove the disc. One should also mention the Italian physician Bonomo, who suggested a similar procedure in 1902. Love introduced the intralaminal-extradural approach for discectomy between 1937 and 1939. Forty years later microsurgery was introduced by pioneers such as Caspar and Yaşargil (1977).

Advances in anesthesia and diagnostic radiology should also be noted. The evolution of antisepsis therapy, along with the development of more effective antibiotic medications, assured safer surgeries. Studies of the biomechanics of the spine continued to be performed and more research data were updated and corrected. . $, 3,69^{3}$

During the same time period, progress was noted in surgical approaches (anterior, posterior, and so forth) and in the use of instrumentation. As a distinct disease entity DDD was crystallizing more than before. King, in 1944, suggested the use of transpedicular screws, but this form of treatment was not used until 1959 by Boucher. Holdsworth and Hardy reported the use of the first fixateur interne in 1953. Dr. Harrington introduced his instrumentation for scoliosis around 1958; it was later used for other conditions. The introduction of the Luque system by Luque and Hall came after this period. Two decades later the Cotrel-Dubousset system was introduced at a time of great need. Several other systems emerged such as the Texas Scottish Rite by Ashman in 1991 and the Moss Miami in 1994 by Shufflebarger. Much of the instrumentation, devices, and approaches that were designed to correct scoliosis also came in handy in the treatment of DDD. Threaded screws, cages, and devices such as the Kaneda system became more popular and applications in lumbar instability and spondylolisthesis flourished. ${ }^{3,8,10,14,19,23}$ Advances in the pathophysiology of DDD followed in a parallel fashion. Diagnostic leaps were made with the advent of CT scanning, myelography with CT scanning, and MR imaging, which helped fortify our understanding of DDD. Although controversial, discography, which was described by Lindblom in 1948, provided a different insight into the pathology of DDD. Magnetic resonance imaging, introduced in the early 1970 s, stands today as an excellent tool for the screening of patients with discogenic pain. With the current development of an upright MR imaging system, some of the limitations of conventional MR imaging will be resolved. One should fairly add that plain radiography 
continues to be an extremely valuable diagnostic tool.

Surgical approaches to treat a ruptured lumbar disc are variable. The most common is the posterior approach, as described earlier. ${ }^{22,24}$ An endoscopic approach was described by Hijikata in 1975 and, before that, chemonucleolysis was started in 1963 by L. Smith and colleagues. With the advent of post-myelography CT scanning, recognition of a far-lateral disc herniation became easier; hence a far-lateral surgical approach was designed. This is an excellent example of the marriage of treatment to diagnostics. During the last few years, several anterior surgical pathways have been described. Taking the aforementioned advances into consideration, DDD remains a great challenge. Today DDD is considered one of the most common spinal disorders. It is thought to cause pain by the mobility that is created and, hence, the segmental instability that is produced. As a result of this understanding of the pathophysiology of the condition, stabilization and fusions have become a popular modality of treatment. Today stabilization is achieved using posterior instrumentation (segmental or nonsegmental), anterior stabilization (cages, devices, and so forth), or a combination of both techniques like that shown in the combined anterior-posterior stabilization. In situ bone fusions are still used, although not as frequently. The history of cages and baskets is quite well documented in the literature and is beyond the scope of this discussion. The use of standalone cages is not favored too greatly and many spine surgeons tend to support these cages with pedicle screws or other devices. ${ }^{2,10,18,21}$

Did the aforementioned advancements resolve all the problems associated with DDD? Are all the patients cured? The answers are clear and many patients still suffer. Outcomes of surgeries in which complications have occurred are very well documented in the literature.

Researchers continue to look at other options and alternatives. The spine is a series of complex joints. When other joints in the human body have degenerated, they are replaced by prosthetic implants. The field of spine arthroplasty started slowly. For example, Froning, in 1975, patented an artificial disc, but never implanted it. Prosthetic disc designs became a challenge with regard to the mobility and elasticity of intervertebral discs. A variety of designs focusing on the viscosity and elasticity of the disc were attempted by Nachemson in 1956, Van Steenbrugghe in 1955, Oyen in 1974, Froning in 1975, Roy-Camille in 1978, Kuntz in 1980, and Edeland in 1981. The list extends to the present with a very extensive list of patents. Parallel to the development of these artificial devices, prosthetics designed to restore motion are also under development. The list of such innovations is too long and beyond the scope of this discussion. Other researchers looked at in situ polymerization devices. Of the hundreds of disc designs, only a few have made it to clinical trials. In the late 1950s, Fernström implanted the first artificial disc. The results of the surgery were not very promising. Hon and his group used a different device in 30 patients, but did not publish their results. The Acroflex design was produced by Steffee and the results were not better than average. In East Germany around the 1980s, Schellnac and Büttner Jans designed the SB Charité device. It was first implanted by Zippel in 1984 and was later aban- doned. The SB Charite III artificial disc was introduced in 1987 and became widely used in Europe. ProDisc was manufactured afterward and is currently still in clinical trials. Along the same line, a Maverick disc is being evaluated. ${ }^{7,21}$ Today devotion to more research continues to define a better prosthesis and the proper indications for its use.

Arthroplasty has several advantages, such as the preservation of function and the decrease of pain. Arthroplasty may avoid the morbidity associated with fusions, but does it solve all the problems of DDD? Although it is still too early to tell, the indications are limited and long-term results have not yet been established. The complex and ambiguous nature of pain caused by DDD and all the other factors associated with it makes it difficult to conceive that one device will solve all the problems. More follow-up reviews and, possibly, different models of the prosthesis, such as one that combines viscoelasticity and motion characteristics at the same time, are needed. Advances in molecular biology and biomaterials that may aid with fusions will also help decrease the rate of morbidity and improve outcomes in other groups of patients. ${ }^{21}$

Surgical innovations and new treatment frontiers will continue to be sought in the quest to find an effective management of DDD. Research in regeneration pathways has begun. A few completed studies indicate a promising future, although progress is slow. Work is focusing on methods to help regenerate the disc. The technology is still primitive and more research and financing are required.

\section{CONCLUSIONS}

Treatment of back pain caused by DDD remains in a dilemma. Medical treatment options benefit a certain group of patients and surgical intervention, which ranges from stabilization to arthroplasty, helps another group of sufferers. Nevertheless, the solutions will not apply for everybody. The complexity of pain from the syndrome of DDD and the fact that we do not fully understand the condition, make it very difficult to devise a common and effective treatment for every patient. It is possible that technological innovations for disc regeneration will produce another modality of treatment for another subgroup of these patients; nevertheless, there will remain a group that receives no good resolution of symptoms. More research and follow-up studies will hopefully produce a better solution to this problem. The "tract" of progress in this area will continue, generating new ideas.

\section{References}

1. Alexander E Jr: The lumbar disk lesions in retrospect and prospect. Surg Neurol 38:315-317, 1992

2. Anonymous: The classic. Disc degeneration and low back pain Ian Macnab. Clin Orthop 208:3-14, 1986

3. Barker FG II: The Massachusetts General Hospital. Early history and neurosurgery to 1939 . J Neurosurg 79:948-959, 1993

4. Benini A, Bonar SK: Andreas Vesalius: 1514-1564. Spine 21: 1388-1393, 1996

5. Brunori A, De Caro GMF, Giuffre R: Chirurgia dell'ernia discale lombare: evoluzione storica. Ann Ital Chir 69:285-293, 1998 


\section{K. J. Chedid and M. K. Chedid}

6. Caspar W: A new surgical procedure for lumbar disc herniation causing less tissue damage through a microsurgical approach. Adv Neurosurg 4:74-80, 1977

7. Griffith SL, Shelokov AP, Büttner-Janz K, et al: A multicenter retrospective study of the clinical results of the LINK SB Charité intervertebral prosthesis. The initial European experience. Spine19:1842-1849, 1994

8. Kaufman HH, Goodrich JT: Byron Stookey: "the old lion"-an unsung giant of neurosurgery. Neurosurgery 40:383-388, 1997

9. Keller T, Holland MC: Some notable American spine surgeons of the 19th century. Spine 22:1413-1417, 1997

10. Knoeller SM, Seifried C: Historical perspective: history of spinal surgery. Spine 25:2838-2843, 2000

11. Marketos SG, Skiadas P: Hippocrates. The father of spine surgery. Spine 24:1381-1387, 1999

12. Marketos SG, Skiadas PK: Galen: a pioneer of spine research. Spine 24:2358-2362, 1999

13. Marketos SG, Skiadas PK: The modern Hippocratic tradition. Some messages for contemporary medicine. Spine 24: 1159-1163, 1999

14. Murphey F: The early days of neurosurgery as I remember them, with emphasis on disc surgery. Neurosurgery 17: 370-372, 1985

15. Naderi S, Acar F, Arda MN: History of spinal disorders and Cerrahiyetül Haniye (Imperial Surgery): a review of a Turkish treatise written by Serefeddin Sabuncuoglu in the 15th century. Historical vignette. J Neurosurg (Spine 3) 96:352-356, 2002

16. Patwardhan RV, Hadley MN: History of surgery for ruptured disk. Neurosurg Clin N Am 12:173-179, 2001

17. Pendl G: History and state of neurosurgery in Austria. Neurosurgery 50:864-869, 2002

18. Pynt J, Higgs J, Mackey M: Historical perspective milestones in the evolution of lumbar spinal postural health in seating. Spine 27:2180-2189, 2002

19. Robinson JS: Sciatica and the lumbar disc syndrome: a historic perspective. South Med J 76:232-238, 1983

20. Schulitz KP, Niggemeyer O: Jacques Calvé. Spine 21:886-890, 1996

21. Szpalski M, Gunzburg R, Mayer M: Spine arthroplasty: a historical review. Eur Spine J 11 (Suppl 2):S65-S84, 2002

22. Waddell G: Low back pain: a twentieth century health enigma. Spine 21:2820-2825, 1996

23. Wright IP: Who is Meyerding? Spine 28:733-735, 2003

24. Videman T, Battié MC, Gibbons LE, et al: Associations between back pain history and lumbar MRI findings. Spine 28: $582-588,2003$

Manuscript received November 17, 2003.

Accepted in final form December 11, 2003.

Address reprint requests to: Mokbel K. Chedid, M.D., 18181

Oakwood Boulevard, Suite 402, Dearborn, Michigan 48124. 